\title{
Effect of ulinastatin, a human urinary protease inhibitor, on heatstroke-induced apoptosis and inflammatory responses in rats
}

\author{
ZHEN TAO $^{1 *}$, FENG-QING HU $^{2 *}$, CHUAN-FEN LI $^{3}$, TAO ZHANG $^{3}$, BING-ZHEN CAO $^{3}$ and LIAN-QI CUI ${ }^{1}$ \\ ${ }^{1}$ Department of Neurology, 401 Hospital of Chinese People's Liberation Army, Qingdao, Shandong 266100; \\ ${ }^{2}$ Department of Cardiothoracic Surgery, Xin Hua Hospital Affiliated to Shanghai Jiao Tong University School of Medicine, \\ Shanghai 200092; ${ }^{3}$ Department of Neurology, General Hospital of Jinan Military Command, \\ Jinan, Shandong 250031, P.R.China
}

Received May 4, 2015; Accepted August 11, 2016

DOI: $10.3892 / \mathrm{etm} .2016 .3926$

\begin{abstract}
Ulinastatin has been demonstrated to protect against heatstroke by reducing cerebral ischemia and damage in rats. In order to extend these observations, apoptosis and systemic inflammatory responses were assessed in rats treated with ulinastatin prior to the initiation of heatstroke. Following the onset of heatstroke, histological analysis revealed that the hippocampal tissues displayed edema and damage. In addition, upregulation of malondialdehyde, inducible nitric oxide synthase (iNOS) and reactive oxygen species and downregulation of superoxide dismutase were observed compared with the respective levels in the control group. Furthermore, TUNEL staining and western blotting assays indicated that heatstroke induced cell apoptosis by increasing the Bax/Bcl-2 ratio and caspase- 3 levels, and upregulating the protein expression levels of nuclear factor- $\kappa \mathrm{B}$, cyclooxygenase- 2 and iNOS. However, the injury induced by heatstroke was significantly inhibited by ulinastatin pretreatment at doses of 5,000 and $10,000 \mathrm{IU} / \mathrm{kg}$. Survival analysis of the rats subjected to heatstroke demonstrated that rats treated with ulinastatin at a dose of $10,000 \mathrm{IU} / \mathrm{kg}$ lived longer than those that did not receive ulinastatin treatment. These observations indicate that ulinastatin may protect against heatstroke by reducing apoptosis and systemic inflammatory responses.
\end{abstract}

Correspondence to: Professor Bing-Zhen Cao, Department of Neurology, General Hospital of Jinan Military Command, 25 Shifan Road, Jinan, Shandong 250031, P.R. China

E-mail: caobzwz@126.com

Professor Lian-Qi Cui, Department of Neurology, 401 Hospital of Chinese People's Liberation Army, 22 Minjiang Road, Qingdao, Shandong 266100, P.R. China

E-mail: cuilq401@126.com

*Contributed equally

Key words: heatstroke, ulinastatin, apoptosis, inflammation responses, nuclear factor- $\kappa \mathrm{B}$, cyclooxygenase- 2

\section{Introduction}

Heatstroke is a life-threatening illness that results from exposure to a high environmental temperature or vigorous exercise, which causes a rapid increase of body temperature (to $>40^{\circ} \mathrm{C}$ ), with consequent abnormalities of the central nervous system, including delerium, convulsions and coma (1). Despite heatstroke being prevalent in individuals in hot climates, it can also affect people in warm climates during heat waves and sporadically in an epidemic form (2). It is predicted that if no effective measures are taken, increasingly hot climates will result in prolonged periods of extremely hot temperatures, with a subsequent increase in mortality rates (3).

Heatstroke is an illness with high morbidity and mortality rates for which no methods of treatment have been clearly defined due to the pathogenesis of cell apoptosis and tissue injury associated with it not being well understood (1). Bouchama et al (4) suggested that tissue injury and cell apoptosis were directly induced by heat. Extreme temperatures are damaging to the majority of cellular structures, and cause functional abnormalities, cell apoptosis and necrosis (5). Rats with moderate hyperthermia have demonstrated accelerated apoptosis, which contributed to cell death (6). However, whether cell death occurs due to apoptosis in patients with heatstroke is not yet understood.

Heatstroke directly induces cell injury as well as the production of large numbers of inflammatory mediators, in addition to cells revealing extensive biological activities that contribute to a systemic inflammatory response and immune dysfunction. In animal models and humans, an increase in circulating pro-inflammatory cytokine levels has been found to induce the development of progression of heatstroke $(4,7,8)$. Cyclooxygenase $(\mathrm{COX})$ is one of the most important pro-inflammatory mediators. In addition, the protein complex nuclear factor $\kappa \mathrm{B}(\mathrm{NF}-\kappa \mathrm{B})$ regulates inflammation by inducing the production of a variety of pro-inflammatory and anti-inflammatory factors (9). In the hypoxia-induced injury of HEI-OC1 cells, it was demonstrated that NF- $\kappa \mathrm{B}$ and hypoxia-inducible factor- 1 were activated, thus causing the upregulation of interleukin-6 release (10). However, whether heatstroke induces an inflammatory response by activating a particular signaling pathway remains unclear. 
Ulinastatin, a typical Kunitz-type protease inhibitor, has been widely used in the treatment of severe heatstroke. Numerous studies have indicated that ulinastatin has protective effects against acute lung injuries caused by endotoxins as well as the mechanical damage resulting from Escherichia coli lipopolysaccharides in rats (11-14). However, there are few studies concerning the use of ulinastatin as an intervention for heatstroke-induced injury in rats (15).

The aim of the present study was to investigate the cellular pathological changes and the associated inflammatory response of hippocampal tissues in rats with heatstroke and the effects of treatment with ulinastatin at different doses. These observations were performed with the aim of identifying the mechanisms of action by which ulinastatin protects against heatstroke in the clinic, as well as the importance of the dose of application.

\section{Materials and methods}

Animals. A total of 48 specific pathogen-free Sprague Dawley male rats (age, 4 weeks; weight, 180-220 g) were purchased from the Shanghai Laboratory Animal Company (Shanghai, China) and housed in the animal facility at $25^{\circ} \mathrm{C}$ (humidity, $60-70 \%$ ) with a 12-h light/dark cycle and free access to food and water. Rats were randomly divided into four groups (12 rats per group). The four groups were as follows: The control group in which the rats were maintained at $25^{\circ} \mathrm{C}$, the heatstroke group where the rats were placed at $42^{\circ} \mathrm{C}$, ulinastatin treatment group 1 where $5,000 \mathrm{IU} / \mathrm{kg}$ ulinastatin was administered $2 \mathrm{~h}$ prior to heating at $42^{\circ} \mathrm{C}$ and ulinastatin treatment group 2 where $10,000 \mathrm{IU} / \mathrm{kg}$ ulinastatin was administered $2 \mathrm{~h}$ prior to heating at $42^{\circ} \mathrm{C}$. The present study was performed in strict accordance with the Guide for the Care and Use of Laboratory Animals of the National Institutes of Health (ninth edition, 2010). The animal use protocol was approved by the Institutional Animal Care and Use Committee of the General Hospital of Jinan Military Command (Jinan, China).

Indicators and methods. Rats were anesthetized via intraperitoneal injection of $3 \%$ sodium pentobarbital $(45 \mathrm{mg} / \mathrm{kg}$; Haoran Biological Technology Co., Ltd., Shanghai, China). Subsequently, the rats, with the exception of those in the control group, were placed into a heating chamber (HD-80TST; Haida Equipment Co., Ltd. Shanghai, China) at $42^{\circ} \mathrm{C}$ with $60 \%$ humidity. Doses of 5,000 and 10,000 IU/kg ulinastatin were administered $2 \mathrm{~h}$ prior to heating to rats in ulinastatin treatment groups 1 and 2, respectively. Following $1 \mathrm{~h}$ of heating, the rats were removed from the heating chamber and returned to their housing conditions.

Histology. Rats were anesthetized with 3\% sodium pentobarbital (40 mg/kg; Sigma-Aldrich; Merck Millipore, Darmstadt, Germany) via intraperitoneal injection prior to cervical dislocation. Hippocampal tissues of rats were removed $1 \mathrm{~h}$ after heating and fixed with $10 \%$ (v/v) neutral-buffered formalin. Specimens were dehydrated and embedded in paraffin and 4-mm tissue sections were cut using a Leica Biosystem Rotary Microtome (Leica Microsystem Nussloch GmbH, Wetzlar, Germany). Next, sections of the tissues were placed on slides, deparaffinized and sequentially stained with hematoxylin and eosin (Richard-Allan
Scientific, Kalamazoo, MI, USA). Under an identical light microscope, the stained tissue sections on slides were analyzed at magnification $\mathrm{x} 200$.

Terminal deoxynucleotidyl transferase-mediated dUTP nick-end labeling (TUNEL) staining. TUNEL staining was performed using an in situ cell death detection kit (POD (11684817910; Roche Diagnostics, Shanghai, China) according to the manufacturer's instructions. The fixed areas of each section were detected by microscopy and the numbers of TUNEL-positive cells were counted at a magnification of x 200 for 30 fields per section.

Biochemical measurements. Hippocampal tissue was homogenized with physiological saline at a ratio of 1:9 (weight/volume) in a homogenizer. The homogenate was then centrifuged at $1,000 \mathrm{x} g$ for $10 \mathrm{~min}$, and the supernatant was collected in order to determine the activity of superoxide dismutase (SOD) and inducible nitric oxide synthase (iNOS) using SOD and iNOS kits. To determine the malondialdehyde (MDA) and reactive oxygen species (ROS) content, MDA and ROS kits were used. The kits used in the present study were purchased from Nanjing Jiancheng Bioengineering Institute (Nanjing, China).

Protein extraction and western blotting. Hippocampus tissues were harvested and lysed on ice for $30 \mathrm{~min}$ in radioimmunoprecipitation assay buffer (Beyotime Institute of Biotechnology, Haimen, China) containing $1 \mathrm{mM}$ phenylmethylsulfonyl fluoride. Total proteins were isolated using radioimmunoprecipitation buffer (Amyjet Scientific, Inc., Wuhan, China) at $10 \mathrm{~min}$ at $95^{\circ} \mathrm{C}$ and centrifuged at $400 \times \mathrm{g}$ at $25^{\circ} \mathrm{C}$ for $10 \mathrm{~min}$. Equal amounts $(50 \mu \mathrm{g})$ of cell lysates was subjected to electrophoresis. Equal amounts of cell lysates were separated by $12 \%$ sodium dodecyl sulfate-polyacrylamide gel electrophoresis and transferred onto nitrocellulose membranes, followed by blocking in fat-free milk overnight at $4^{\circ} \mathrm{C}$. The blots were incubated with primary antibodies targeting Bax (1:1,000; sc-493), Bcl-2 (1:200; sc-492; both Santa Cruz Biotechnology, Inc., Dallas, TX, USA), caspase-3 (1:5,000; ab32351; Abcam, Cambridge, MA, USA), COX-2 (1:1,000; 12282; Cell Signaling Technology, Inc., Danvers, MA, USA), iNOS (1:800; ab3523; Abcam), NF- $\mathrm{B}$ p65 (1:1,000; 6956), H3 (1:1,000; 4499) and glyceraldehyde 3-phosphate dehydrogenase $(1: 1,500 ; 1574$; all Cell Signaling Technology, Inc.) at $4^{\circ} \mathrm{C}$ overnight. Following washing, the membranes were subsequently incubated with horseradish peroxidase-conjugated goat anti-rabbit IgG (1:1,000; A0208) and goat anti-mouse IgG (1:1,000; A0216; both Beyotime Institute of Biotechnology) secondary antibodies for $1 \mathrm{~h}$ at $37^{\circ} \mathrm{C}$, and were washed three times with Tris-buffered saline with Tween 20 (Amresco LLC, Solon, OH, USA). Chemiluminescence detection was conducted using Western Lightning Chemiluminescence Reagent Plus (PerkinElmer, Inc., Waltham, MA, USA) and signals were quantified by densitometry (Quantity One software, version 4.62; Bio-Rad Laboratories, Inc., Hercules, CA, USA).

Statistical analysis. The data were presented as the mean value \pm standard deviation. Paired, two-tailed Student's t-test and one-way analysis of variance were used to analyze the significance of a difference between groups. Survival analysis was performed by the Kaplan-Meier method, and subjected to 
A

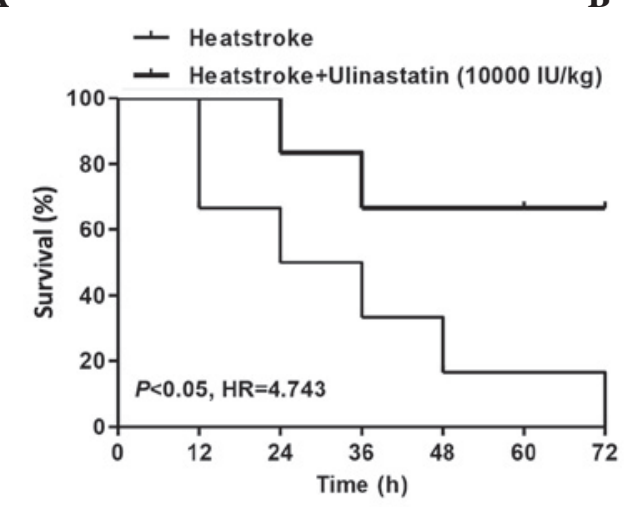

B

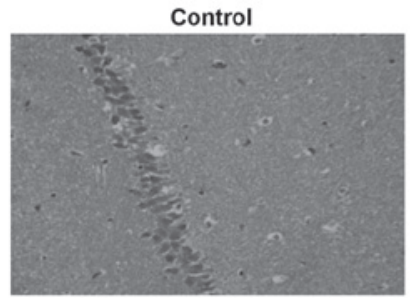

Ulinastatin (5000 IU/kg)

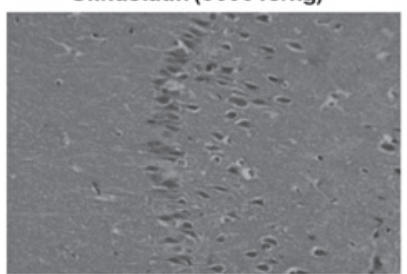

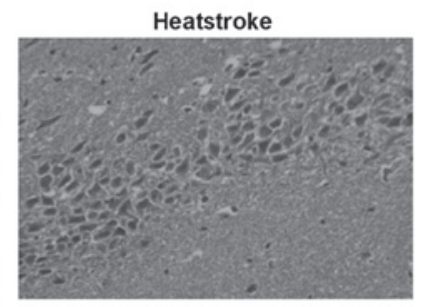

Ulinastatin (10000 IU/kg)

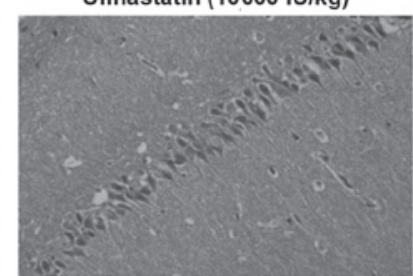

Figure 1. Survival analysis of rats with heatstroke, and hematoxylin and eosin staining of the rats' hippocampal tissue. (A) Survival analysis revealed that untreated rats with heatstroke had a poor prognosis compared with those that were pretreated with ulinastatin. (B) Ulinastatin treatment at doses of 5,000 and $10,000 \mathrm{IU} / \mathrm{kg}$ attenuated the edema and loss of cell nuclei in rats induced by heatstroke (magnification, x200). HR, hazard ratio.

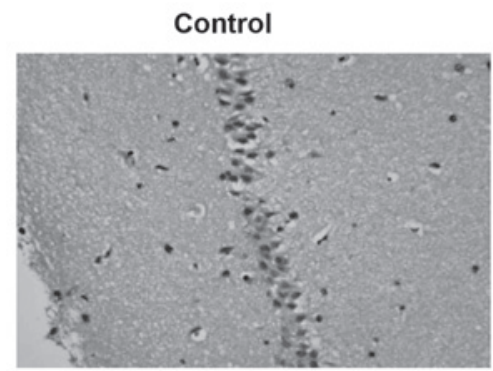

Ulinastatin $(5000 \mathrm{IU} / \mathrm{kg})$

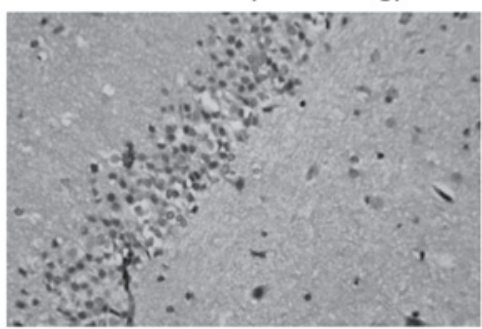

Heatstroke

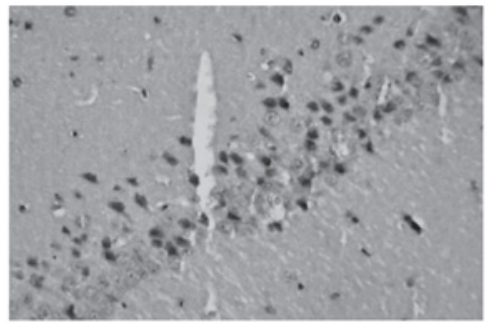

Ulinastatin $(10000 \mathrm{lU} / \mathrm{kg})$

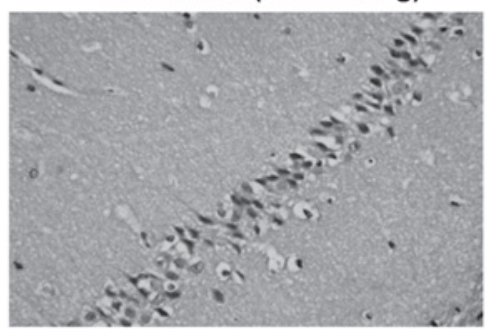

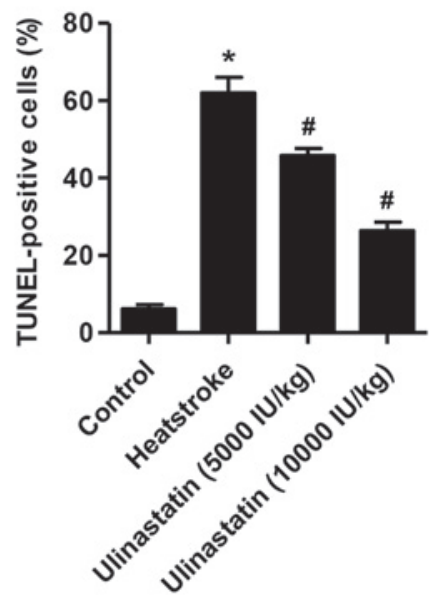

Figure 2. Effect of ulinastatin on heatstroke-induced cell apoptosis in rats. Ulinastatin treatment at the doses of 5,000 and 10,000 IU/kg attenuated the heatstroke-induced increase in the number apoptotic cells of the hippocampal nucleus. Representative images of TUNEL staining (magnification x200) and quantified results are shown. Data are presented as the mean + standard deviation ( $\mathrm{n}=12 /$ group). ${ }^{*} \mathrm{P}<0.01 \mathrm{vs}$. the control and ${ }^{\#} \mathrm{P}<0.01$ vs. the heatstroke group. TUNEL, terminal deoxynucleotidyl transferase-mediated dUTP nick-end labeling.

the log rank test. Data are presented as the mean + standard deviation ( $\mathrm{n}=12$ /group). $\mathrm{P}<0.05$ was considered to indicate a statistically significant difference.

\section{Results}

Ulinastatin protects rats against heatstroke-induced injury. The survival times of rats subjected to heatstroke with and without ulinastatin treatment were compared. The cumulative survival rate was significantly lower in the heatstroke group than in the rats with heatstroke treated with ulinastatin at a dose of 10,000 IU/kg (Fig. 1A; P<0.05). These results indicate that ulinastatin could improve the prognosis of rats with heatstroke. Following the onset of heatstroke, the damage to hippocampal neurons was greater in the rats of the heatstroke group compared with the control group. Histological examination revealed that heatstroke caused edema and disappearance of cell nuclei in the hippocampus of rats (Fig. 1B). However, ulinastatin treatment at doses of 5,000 and $10,000 \mathrm{IU} / \mathrm{kg}$ inhibited the effects induced by heatstroke and thus exhibited neuroprotective effects.

Ulinastatin protects rats against heatstroke-induced apoptosis. Fig. 2 summarizes the effects of heatstroke on the number of TUNEL-positive cells in the hippocampus of the control, heatstroke and ulinastatin-treated rats. Following the onset of heatstroke, the number of TUNEL-positive cells of the hippocampus was increased in the heatstroke group compared with the control group (Fig. 2; P<0.01). However, the increase in the number of TUNEL-positive cells in the hippocampus 
A

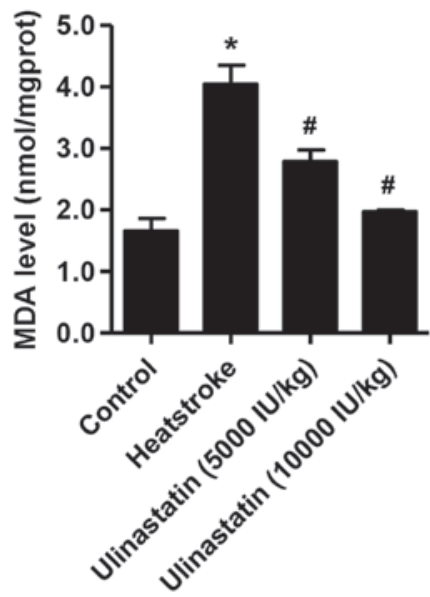

C

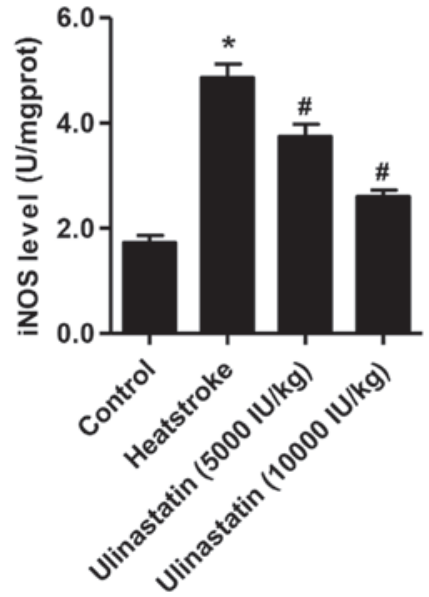

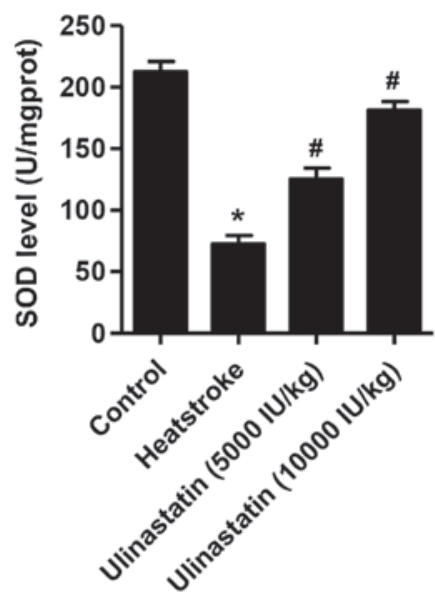

D

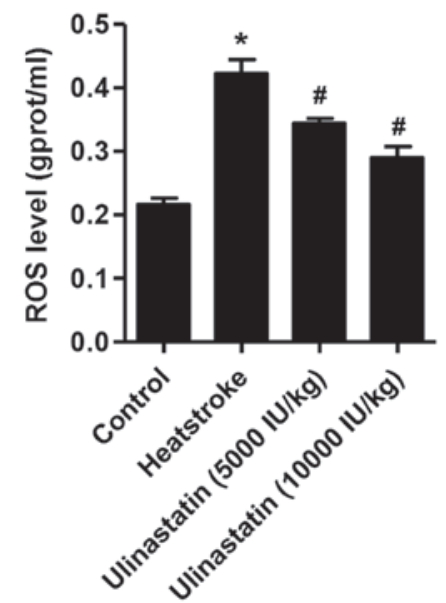

Figure 3. Biochemical measurements in heatstroke-induced rats. (A) MDA, (B) SOD, (C) iNOS and (D) ROS levels. Ulinastatin at 5,000 and 10,000 IU/kg attenuated the upregulation of MDA, iNOS and ROS and downregulation of ROS levels induced by heatstroke. Data are presented as the mean + standard deviation ( $\mathrm{n}=12$ /group). ${ }^{*} \mathrm{P}<0.01$ vs. the control and ${ }^{*} \mathrm{P}<0.01$ vs. the heatstroke group. MDA, malondialdehyde; SOD, superoxide dismutase; iNOS, inducible nitric oxide synthase; ROS, reactive oxygen species.

of rats with heatstroke was greatly attenuated by ulinastatin at doses of 5,000 and 10,000 IU/kg $(\mathrm{P}<0.01)$.

Ulinastatin treatment downregulates MDA, iNOS and ROS levels but upregulates the SOD level in rats with heatstroke. Fig. 3 shows the levels of MDA, SOD, iNOS and ROS among the three experimental groups and the control group. Compared with the control group, following the onset of heatstroke, the rats had higher levels of MDA, iNOS and ROS $(\mathrm{P}<0.01)$ and a lower level of SOD $(\mathrm{P}<0.01)$. However, compared with the heatstroke group, rats treated with ulinastatin at doses of 5,000 and 10,000 IU/kg had significantly lower levels of MDA, iNOS and ROS, and higher levels of SOD following the induction of heatstroke $(\mathrm{P}<0.01)$.

Ulinastatin decreases the Bax/Bcl-2 ratio and caspase- 3 levels of rats with heatstroke. The Bcl-2 family contains pro-apoptotic (e.g., Bax) and anti-apoptotic (e.g., Bcl-2) proteins for the regulation of apoptosis and death progression (16-18). Western blot analysis was performed to detect Bcl-2, Bax and caspase-3 levels in heatstroke rats. Heatstroke resulted in a significant reduction in the level of the anti-apoptotic protein Bcl-2 accompanied by increases in the levels of the pro-apoptotic proteins Bax and caspase-3 compared with those in the control group (Fig. 4; $\mathrm{P}<0.01$ ). However, the rats treated with ulinastatin at doses of 5,000 and $10,000 \mathrm{IU} / \mathrm{kg}$ had lower $\mathrm{Bax} / \mathrm{Bcl}-2$ ratios and caspase- 3 levels compared with the heatstroke rats without ulinastatin treatment. These data indicate that heatstroke increases the Bax/Bcl-2 ratio and caspase-3 levels, which may contribute to the increase of cell apoptosis, and ulinastatin attenuates the apoptosis in heatstroke rats.

Ulinastatin inhibits NF- $\kappa B$ p65, COX-2 and iNOS levels of rats with heatstroke. Rats exposed to heat for $1 \mathrm{~h}$ exhibited significantly increased the intranuclear NF- $\kappa$ B p65 level compared with the control, and since the expression of $\mathrm{NF}-\kappa \mathrm{B}$ p65 is the central step of $N F-\kappa B$ activation, this indicated that heatstroke may cause $\mathrm{NF}-\kappa \mathrm{B}$ activation $(\mathrm{P}<0.01$; Fig. $5 \mathrm{~A})$. Pretreatment of rats with 5,000 or $10,000 \mathrm{IU} / \mathrm{kg}$ ulinastatin $2 \mathrm{~h}$ prior to heat exposure significantly suppressed NF- $\mathrm{NB}$ p65 subunit nuclear translocation $(\mathrm{P}<0.01)$. Furthermore, pretreatment with ulinastatin abrogated not only heatstroke-induced COX-2 upregulation but also the protein level of iNOS (Fig. 5B; $\mathrm{P}<0.01)$. These observations indicate that the protective effects of ulinastatin against inflammatory response caused by heatstroke are associated with the attenuation of $N F-\kappa B$ activation in rats. 


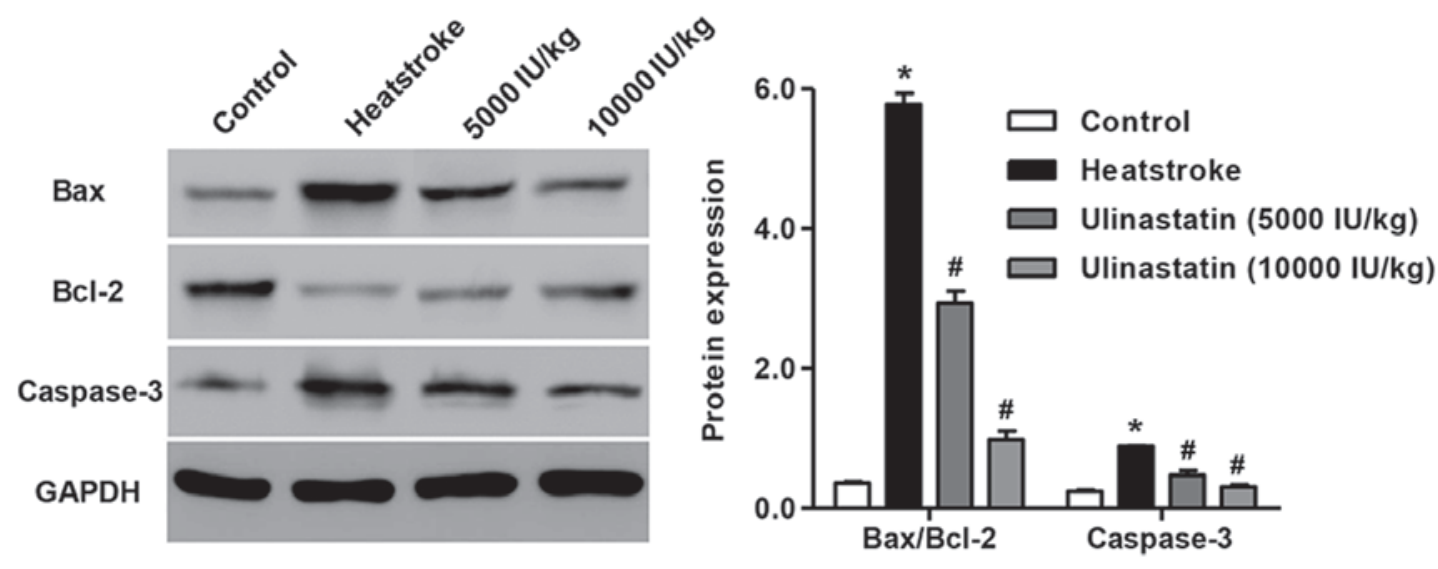

Figure 4. Expression of Bax, Bcl-2 and caspase-3 in heatstroke-induced rats. Representative western blots and quantified levels (relative to GAPDH) are shown. Ulinastatin at 5,000 and 10,000 IU/kg attenuated the increase of the Bax/Bcl-2 expression ratio and caspase-3 level in rats induced by heatstroke. Data are presented as the mean + standard deviation ( $\mathrm{n}=12 /$ group). ${ }^{*} \mathrm{P}<0.01$ vs. the control and ${ }^{\#} \mathrm{P}<0.01$ vs. the heatstroke group. Bcl-2, $\mathrm{B}-$ cell lymphoma $2 ; \mathrm{Bax}, \mathrm{Bcl} 2$ associated X protein; GAPDH, glyceraldehyde 3-phosphate dehydrogenase.
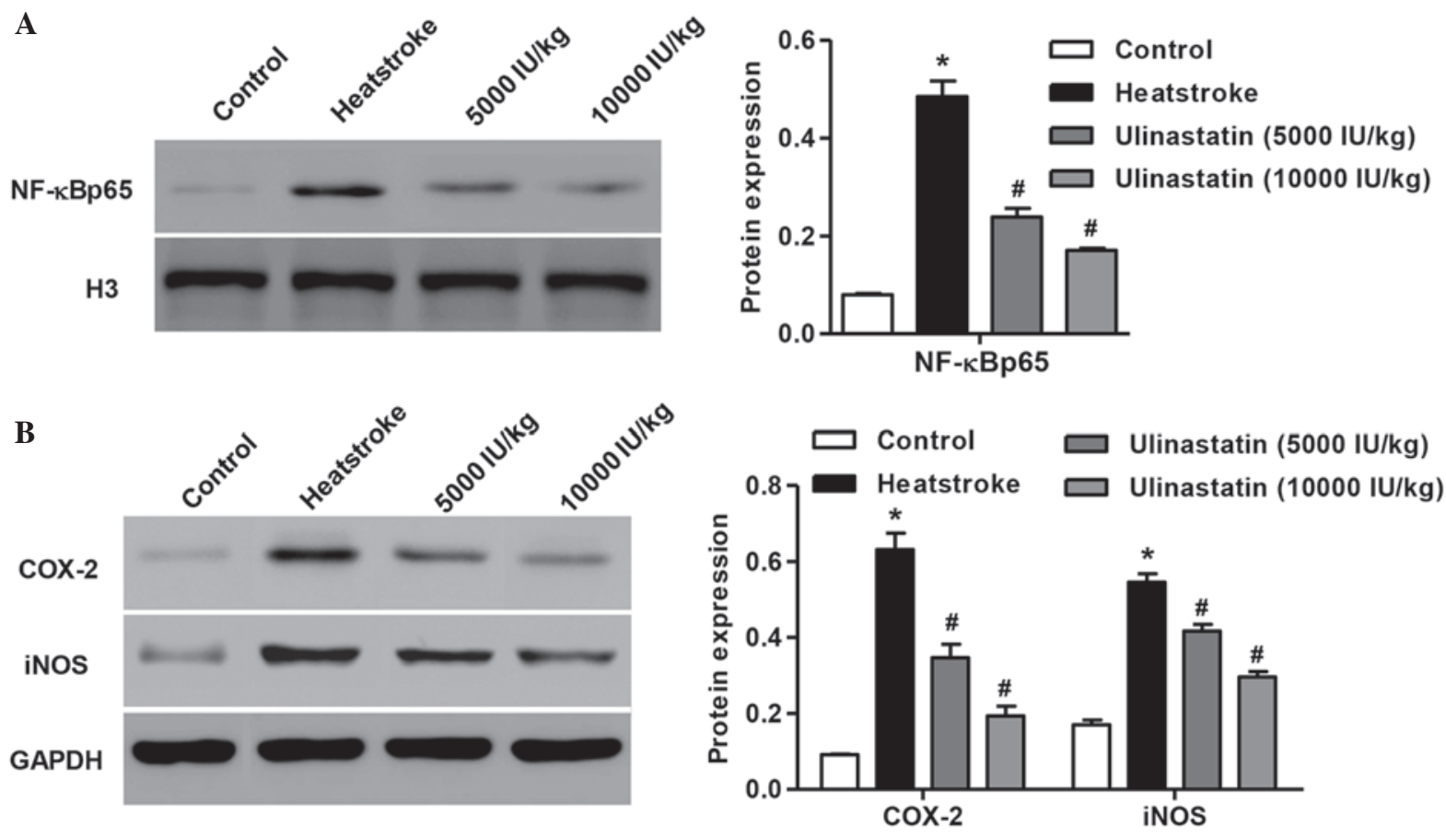

Figure 5. Effect of ulinastatin on heatstroke-induced inflammation responses. Representative western blots and quantified levels (relative to GAPDH) are shown. Ulinastatin at doses of 5,000 and 10,000 IU/kg attenuated the increase of the protein expression of (A) NF-kBp65, (B) COX-2 and iNOS in rats induced by heatstroke. Data are presented as the mean + standard deviation ( $\mathrm{n}=12 /$ group). ${ }^{*} \mathrm{P}<0.01$ vs. the control and ${ }^{\#} \mathrm{P}<0.01$ vs. the heatstroke group. NF- $\mathrm{kB}$, nuclear factor $\mathrm{\kappa B}$; COX-2, cyclooxygenase-2; iNOS, inducible nitric oxide synthase; GAPDH, glyceraldehyde 3-phosphate dehydrogenase.

\section{Discussion}

The aim of the present study was to elucidate the pathogenic and molecular mechanisms that underlie tissue injury and cell apoptosis in heatstroke using a rat model. One of the principal observations of the present study was that the survival time of heatstroke rats pretreated with ulinastatin at a dose of $10,000 \mathrm{IU} / \mathrm{kg}$ was notably longer than that of heatstroke rats without ulinastatin treatment. Furthermore, heatstroke induced edema and the loss of nuclei from hippocampal neurons in rats and induced cell apoptosis by increasing the $\mathrm{Bax} / \mathrm{Bcl}-2$ ratio and caspase-3 protein levels. Secondly, heatstroke also upregulated MDA, iNOS and ROS levels but downregulated SOD levels, and induced inflammatory responses via increasing the expression of NF- $\mathrm{kB}$ p $65, \mathrm{COX}-2$ and iNOS. Thirdly, pretreatment with ulinastatin significantly attenuated the injury induced by heatstroke.

There are numerous different mechanisms by which heatstroke induces damage in the body (19). Previous studies have demonstrated that high temperature and humidity are two principal factors in the induction of a hyperthermic animal model of heatstroke $(4,20)$. The histological examination conducted in the present study revealed that the hippocampal neurons of the rats in the heatstroke group presented extensive patholog- 
ical changes compared with those of the control group. Light microscopy revealed the presence of edema in and the loss of nuclei from hippocampal neurons in the heatstroke-induced rats (Fig. 1B). However, the brain injury induced by heatstroke in the rats was attenuated by ulinastatin treatment. This is consistent with a previous study in which Qin et al (21) identified that the early application of ulinastatin relieved edema in the lung tissue of rats subjected to hyperthermia.

Another interesting observation of the present study was the critical role of apoptosis as a mechanism of cell death in heatstroke. An increase in apoptosis was observed in heatstroke-induced rats using the TUNEL assay (Fig. 2). These observations were similar to those in baboons subjected to heatstroke (22). Furthermore, to further understand the mechanism of cell apoptosis, the expression of Bax, Bcl-2 and caspase- 3 levels in heatstroke-induced rats was detected. As shown in Fig. 4, the $\mathrm{Bax} / \mathrm{Bcl}-2$ ratio and the protein level of caspase-3 were increased in heatstroke-induced rats. Importantly, heatstroke rats treated with ulinastatin revealed a reduction in the number of TUNEL-positive cells and an attenuated increase of $\mathrm{Bax} / \mathrm{Bcl}-2$ ratio and caspase -3 protein level, compared with rats without ulinastatin treatment. These results demonstrate that apoptosis is associated with the pathophysiology of heat stress. In the present study, the results also demonstrated that ulinastatin suppressed the MDA, iNOS and ROS upregulation and SOD downregulation induced by heatstroke in rats (Figs. 3 and 5). These data are consistent with results of other studies, which demonstrated that ulinastatin administration inhibited the increase of MDA, iNOS and ROS levels, and increased SOD levels in various types of damage in animal models (23-26).

In humans and animals subjected to heat stress, an inflammatory response has been shown to contribute to the damage of various tissues and organs (8). COX-2, a pro-inflammatory mediator, promotes the production of a number of inflammatory factors in multiple cells and tissues. Tsutakawa et al (27) identified that COX-2 mRNA expression is upregulated in rat skin with ischemia/reperfusion (I/R)-induced lesions, and that NS-398, a COX-2 inhibitor, protects against nicotine exacerbated I/R-triggered skin apoptosis and necrosis. The present study indicated that exposure of rats to heat caused the expression level of COX-2 to increase, and that pretreatment with ulinastatin for $2 \mathrm{~h}$ suppressed heatstroke-induced COX-2 upregulation. $\mathrm{NF}-\kappa \mathrm{B}$ is an inducible transcription factor that increases COX-2 expression levels (28). The p65 protein is the most abundant subunit of NF- $\kappa \mathrm{B}$, with p50, p52, REL and REL-B being the others, and promotes the activation of $N F-\kappa B$. A previous study identified that ulinastatin inhibited the translocation of the NF- $\mathrm{NB}$ p65 subunit from the cytoplasm to the nucleus in rats with smoke inhalation-induced injury (29). In agreement with this previous study, the exposure of rats to heat in the present study resulted increased $N F-\kappa B$ p 65 expression, which was notably reversed by pretreatment with ulinastatin. The results demonstrate the association between $N F-\kappa B$ and inflammation, and suggest that ulinastatin protects against heatstroke-induced inflammatory responses by inhibiting the $\mathrm{NF}-\kappa \mathrm{B} / \mathrm{COX}-2$ pathway in rats.

Overall, to the best of our knowledge the present study is the first to suggest that ulinastatin exhibits a protective effect against brain injury and inflammatory responses induced by heatstroke through the inhibition of cell apoptosis and blockage of the NF- $\mathrm{B} / \mathrm{COX}-2$ pathway in rats. Furthermore, the present study provides novel evidence concerning the mechanism by which ulinastatin reduces heatstroke-induced brain injury. Therefore, administration of ulinastatin may be a novel therapeutic strategy for brain injury induced by heatstroke.

\section{References}

1. Bouchama A and Knochel JP: Heat stroke. N Engl J Med 346: 1978-1988, 2002.

2. Vandentorren S, Bretin P, Zeghnoun A, Mandereau-Bruno L, Croisier A, Cochet C, Ribéron J, Siberan I, Declercq B and Ledrans M: August 2003 heat wave in France: Risk factors for death of elderly people living at home. Eur J Public Health 16: 583-591, 2006.

3. Patz JA, Campbell-Lendrum D, Holloway T and Foley JA: Impact of regional climate change on human health. Nature 438: 310-317, 2005.

4. Bouchama A, Roberts G, Al Mohanna F, El-Sayed R, Lach B, Chollet-Martin S, Ollivier V, Al Baradei R, Loualich A, Nakeeb S, et al: Inflammatory, hemostatic and clinical changes in a baboon experimental model for heatstroke. J Appl Physiol (1985) 98: 697-705, 2005.

5. Buckley IK: A light and electron microscopic study of thermally injured cultured cells. Lab Invest 26: 201-209, 1972.

6. Sakaguchi Y, Stephens LC, Makino M, Kaneko T, Strebel FR, Danhauser LL, Jenkins GN and Bull JM: Apoptosis in tumors and normal tissues induced by whole body hyperthermia in rats. Cancer Res 55: 5459-5464, 1995.

7. Chen CM, Hou CC, Cheng KC, Tian RL, Chang CP and Lin MT: Activated protein C therapy in a rat heat stroke model. Crit Care Med 34: 1960-1966, 2006.

8. Lu KC, Wang JY, Lin SH, Chu P and Lin YF: Role of circulating cytokines and chemokines in exertional heatstroke. Crit Care Med 32: 399-403, 2004.

9. Abd-El-Aleem SA, Ferguson MW, Appleton I, Bhowmick A, McCollum CN and Ireland GW: Expression of cyclooxygenase isoforms in normal human skin and chronic venous ulcers. J Pathol 195: 616-623, 2001.

10. Jeong HJ, Hong SH, Park RK, Shin T, An NH and Kim HM: Hypoxia-induced IL- 6 production is associated with activation of MAP kinase, HIF-1 and NF-kappaB on HEI-OC1 cells. Hear Res 207: 59-67, 2005.

11. Inoue K, Takano H, Sato H, Yanagisawa R and Yoshikawa T: Protective role of urinary trypsin inhibitor in lung expression of proinflammatory cytokines accompanied by lethal liver injury in mice. Immunopharmacol Immunotoxicol 31: 446-450, 2009.

12. Wang W, Huang W, Chen S, Li Z, Wang W and Wang M: Changes of tumor necrosis factor-alpha and the effects of ulinastatin injection during cardiopulmonary cerebral resuscitation. J Huazhong Univ Sci Technolog Med Sci 24: 269-271, 2004.

13. Bae HB, Jeong CW, Li M, Kim HS and Kwak SH: Effects of urinary trypsin inhibitor on lipopolysaccharide-induced acute lung injury in rabbits. Inflammation 35: 176-182, 2012.

14. Zhang X, Liu F, Liu H, Cheng H, Wang W, Wen Q and Wang Y: Urinary trypsin inhibitor attenuates lipopolysaccharide-induced acute lung injury by blocking the activation of p38 mitogen-activated protein kinase. Inflamm Res 60: 569-575, 2011.

15. Zheng CY, Zhang W and Liang YG: Inflammatory factor level and ulinastatin intervention in the early stage of heat stress in rats. J Med Postgraduates 24: 25-28, 2011.

16. Adams JM and Cory S: Life-or-death decisions by the Bcl-2 protein family. Trends Biochem Sci 26: 61-66, 2001.

17. Hetz C: BCL-2 protein family: Essential regulators of cell death. Preface. Adv Exp Med Biol 687: vii-viii, 2010.

18. Reed JC: Regulation of apoptosis by bcl-2 family proteins and its role in cancer and chemoresistance. Curr Opin Oncol 7: 541-546, 1995.

19. Hernandez JF, Secrest JA, Hill L and McClarty SJ: Scientific advances in the genetic understanding and diagnosis of malignant hyperthermia. J Perianesh Nurs 24: 19-34, 2009.

20. Wang HM, Bodenstein M and Markstaller K: Overview of the pathology of three widely used animal models of acute lung injury. Eur Surg Res 40: 305-316, 2008. 
21. Qin ZS, Tian P, Wu X, Yu HM and Guo N: Effects of ulinastatin administered at different time points on the pathological morphologies of the lung tissues of rats with hyperthermia. Exp Ther Med 7: 1625-1630, 2014.

22. Roberts GT, Ghebeh H, Chishti MA, Al-Mohanna F, El-Sayed R, Al-Mohanna F and Bouchama A: Microvascular injury, thrombosis, inflammation and apoptosis in the pathogenesis of heatstroke: A study in baboon model. Arterioscl Throm Vasc Biol 28: 1130-1136, 2008.

23. Xu M, Wen X, Chen S, An X and Xu H: Addition of ulinastatin to preservation solution promotes protection against ischemia-reperfusion injury in rabbit lung. Chin Med J (Engl) 124: 2179-2183, 2011.

24. Hua G, Haiping Z, Baorong $\mathrm{H}$ and Dingjun $\mathrm{H}$ : Effect of ulinastatin on the expression of iNOS, MMP-2 and MMP-3 in degenerated nucleus pulposus cells of rabbits. Connect Tissue Res 54: 29-33, 2013.
25. Tong Y, Tang Z, Yang T, Yang Y, Yang L, Shen W and Chen W: Ulinastatin preconditioning attenuates inflammatory reaction of hepatic ischemia reperfusion injury in rats via high mobility group box 1 (HMGB1) inhibition. Int J Med Sci 11: 337-343, 2014.

26. Yu JB and Yao SL: Protective effects of hemin pretreatment combined with ulinastatin on septic shock in rats. Chin Med J (Engl) 121: 49-55, 2008.

27. Tsutakawa S, Kobayashi D, Kusama M, Moriya T and Nakahata N: Nicotine enhances skin necrosis and expression of inflammatory mediators in a rat pressure ulcer model. Brit J Dermatol 161: 1020-1027, 2009.

28. Kang YJ, Wingerd BA, Arakawa $\mathrm{T}$ and Smith WL: Cyclooxygenase-2 gene transcription in a macrophage model of inflammation. J Immunol 177: 8111-8122, 2006.

29. Qiu X, Ji S, Wang J, Li H, Xia T, Pan B, Xiao S and Xia Z: The therapeutic efficacy of Ulinastatin for rats with smoking inhalation injury. Int Immunopharmacol 14: 289-295, 2012. 